\title{
Spontaneous Fracture of Plastic Stents: A Rare Complication of Long-Term Indwelling Transmural Endoprosthesis
}

\author{
Surinder Singh Rana ${ }^{1}$ Ravi Sharma ${ }^{1}$ Rajesh Gupta ${ }^{2}$ \\ ${ }^{1}$ Departments of Gastroenterology, Post Graduate Institute of \\ Medical Education and Research (PGIMER), Chandigarh, India \\ ${ }^{2}$ Surgical Gastroenterology, Post Graduate Institute of Medical \\ Address for correspondence Dr Surinder Singh Rana, MD, \\ D.M, Professor, Department of Gastroenterology, PGIMER, \\ Chandigarh-160 012, India (e-mail: drsurinderrana@gmail.com).
} Education and Research (PGIMER), Chandigarh, India

J Digest Endosc 2021;12:43-45.

\begin{abstract}
Keywords

- walled-off necrosis

- stent

- pancreatitis

- pseudocyst

Permanent indwelling transmural stents is a safe and effective strategy for prevention of recurrence of pancreatic fluid collections following successful endoscopic transmural drainage of walled-off necrosis (WON) with coexistent disconnected pancreatic duct syndrome (DPDS). Long-term studies have shown this strategy to be safe and effective with rarely reported adverse effects. In this case series, we describe three patients of successfully treated WON and DPDS presenting later with a rare complication of permanent indwelling transmural plastic stents.
\end{abstract}

\section{Introduction}

Disconnected pancreatic duct syndrome (DPDS) is an important but often ignored complication of acute necrotizing pancreatitis (ANP). It develops due to the necrosis of main pancreatic duct $(\mathrm{PD})$, leading on to disconnection between the viable upstream pancreatic parenchyma and the gastrointestinal (GI) tract. ${ }^{1}$ This disconnected and functional segment of pancreas continues to secrete pancreatic juice that is not drained into the GI tract. In a patient with successfully treated walled-off necrosis (WON) and endoscopic transmural drainage, removal of transmural stents can lead to recurrence of pancreatic fluid collections (PFC) because of ongoing secretion of pancreatic juice from the disconnected segment of pancreas. ${ }^{2,3}$ This risk of PFC recurrence is highest in the first year following successful resolution. ${ }^{2}$ This problem of recurrence of PFC can be successfully mitigated by leaving transmural stents in situ indefinitely. ${ }^{3,4}$

Theoretically, leaving plastic stents for an indefinite period in situ can be associated with significant concerns such as infection, bleeding, stent migration, stent fracture, GI perforation or intestinal obstruction due to stent migration. ${ }^{4}$ However, long-term studies have shown that this strategy of leaving transmural stents for an indefinite period following resolution of WON associated with DPDS is not associated with major

published online

May 9, 2020
DOI https://doi.org/

$10.1055 / \mathrm{s}-0040-1714044$

ISSN 0976-5042. complications. ${ }^{4-6}$ In this case series, we describe three patients of WON and DPDS with permanent indwelling polyethylene transmural stents developing a rare and unreported complication of fracture of plastic stents. In all these three patients, this complication could be managed endoscopically.

\section{Case 1}

A 43-year-old man was referred to our Centre for endoscopic ultrasound (EUS)-guided drainage of symptomatic WON that developed following alcohol-related ANP. The patient underwent EUS-guided transmural drainage with multiple plastic stents. Following resolution, two $7 \mathrm{Fr} 3 \mathrm{~cm}$ double pigtail plastic stents were left in situ (-Fig. 1), as magnetic resonance cholangiopancreatography (MRCP) revealed a disconnected PD. The patient presented 9 months later with recurrence of abdominal pain and normal serum amylase and lipase. Abdominal X-ray revealed fractured plastic transmural stents ( $\boldsymbol{- F i g .} \mathbf{2}$ a) and $\mathrm{CT}$ revealed fractured plastic stents with the fractured tip being buried in the stomach wall ( - Fig. $\mathbf{2}$ b). Upper GI endoscopy showed the tip of one of the fractured stents, whereas the other stent could not be localized ( - Fig. $\mathbf{3}$ ). The fractured stent was removed using rat-tooth forceps

(C)2020. Society of Gastrointestinal Endoscopy of India.

This is an open access article published by Thieme under the terms of the Creative Commons Attribution-NonDerivative-NonCommercial-License, permitting copying and reproduction so long as the original work is given appropriate credit. Contents may not be used for commercial purposes, or adapted, remixed, transformed or built upon. (https://creativecommons.org/licenses/by-nc-nd/4.0/).

Thieme Medical and Scientific Publishers Pvt. Ltd. A-12, 2nd Floor, Sector 2, Noida-201301 UP, India 


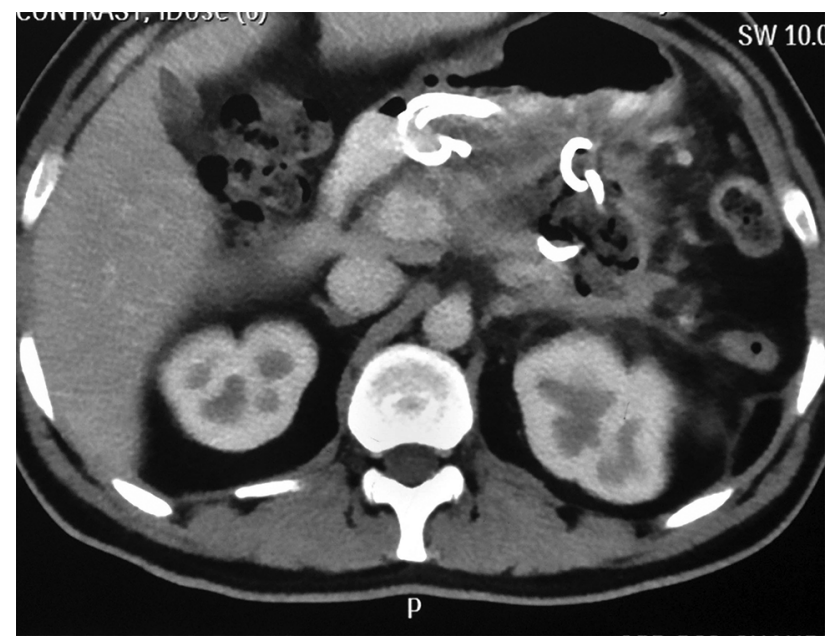

Fig. 1 Contrast-enhanced CT abdomen: Two $7 \mathrm{Fr} 3 \mathrm{~cm}$ double pigtail plastic stents seen in situ after resolution of WON. Abbreviation: WON, walled-off necrosis.

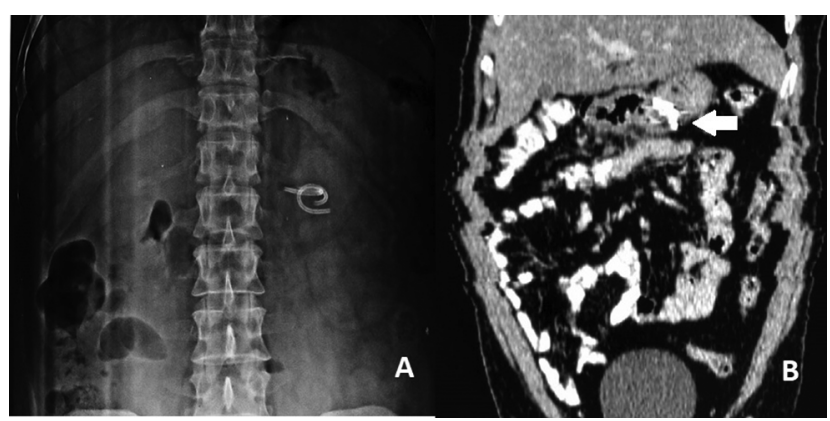

Fig. 2 (a) Abdominal X-ray: Fractured plastic transmural stents with external pigtail of both the stents fractured and migrated. (b) CT abdomen: Fractured plastic stents with fractured tip being buried in the stomach wall (arrow).

and the entangled other fractured stent could also be retrieved along with it ( $\boldsymbol{- F i g .} 3$ [inset]). Following this, the pain resolved, and the patient remained asymptomatic over a follow-up period of 4 months.

\section{Case 2}

A 38-year-old man with WON secondary to alcohol-related ANP was successfully treated with EUS-guided transmural drainage. Thereafter, two $7 \mathrm{Fr} 3 \mathrm{~cm}$ double pigtail plastic stents were left in situ as endoscopic retrograde cholangiopancreatography (ERCP) revealed complete cutoff of main PD. Two years later, he presented with abdominal pain and abdominal X-ray revealed fractured plastic transmural stents. Upper GI endoscopy revealed fracture external pigtail of both the stents. The tip of both the fractured stents was visible in the stomach on endoscopy, and both the fractured stents were removed by grasping the ends of the fractured stents using rat-tooth forceps, leading to resolution of pain. The patient is asymptomatic over a follow-up period of 2 months.

\section{Case 3}

A 42-year-old female with WON secondary to gall stone ANP was successfully treated with EUS-guided transmural drainage.

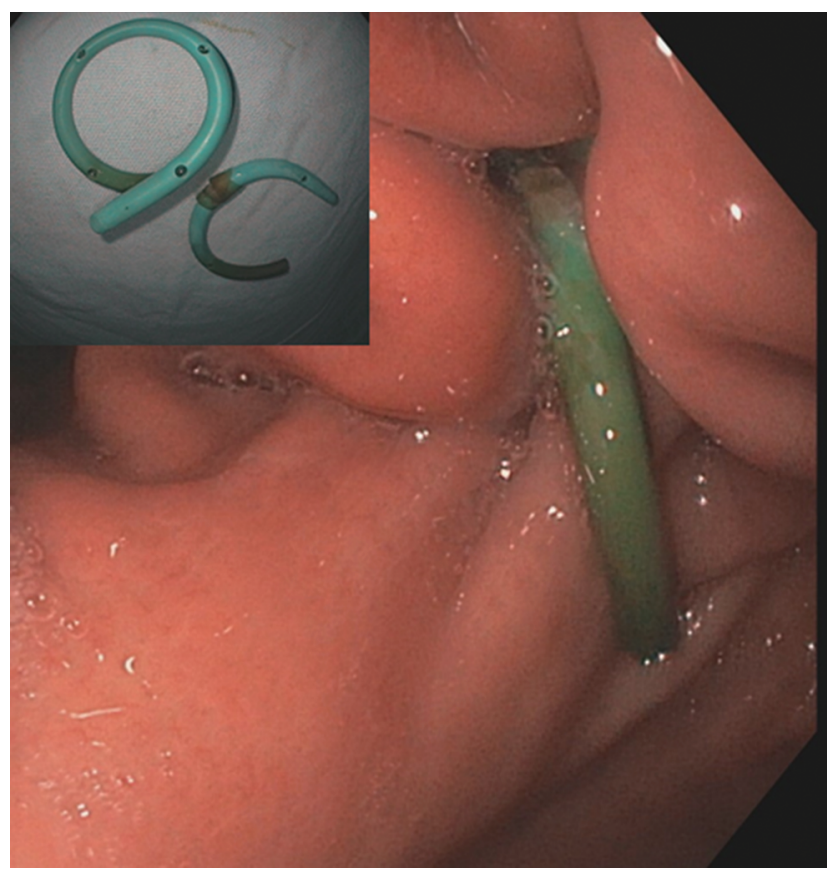

Fig. 3 Upper Gl endoscopy: Tip of one of the fractured stents could be localized, whereas the other stent could not be localized. (Inset: Both the stents after endoscopic removal). Abbreviation: $\mathrm{Gl}$, gastrointestinal.

Thereafter, a $10 \mathrm{Fr} 3 \mathrm{~cm}$ double pigtail plastic stent was left in situ as ERCP revealed DPDS. Patient underwent laparoscopic cholecystectomy and was asymptomatic for the next 3 years. Thereafter, she presented with asymptomatic passage of a part of plastic stent in the stool. Abdominal X-ray revealed fractured transmural stent. Upper GI endoscopy revealed fracture of external pigtail of the stent. The tip of the fractured stent was visible in the stomach on endoscopy, and the fractured stent was removed by grasping the end of the fractured stent using rat-tooth forceps. The patient is asymptomatic over a follow-up period of 1 year.

\section{Discussion}

To reduce the risk of recurrence of PFC in patients with DPDS, transmural stents are left in situ permanently, following successful transmural drainage, so that the disconnected pancreas continues to have an alternative internal drainage route. ${ }^{4-6}$ Despite concerns of leaving a plastic prosthesis indefinitely, studies have shown that this strategy of leaving stents for an indefinite period is safe and effective. There are only few reports of significant adverse effects of permanent indwelling stents. The rare adverse events reported are intestinal obstruction due to external migration of the stents, colonic perforation, pancreatic parenchymal calcification, asymptomatic external migration of plastic stents, and morphological changes resembling chronic pancreatitis in the disconnected pancreas. $5,7,8$

Fracture of permanent indwelling transmural stents have not been reported previously. In our study, fracture of stents occurred 9 months to 36 months after their placement. Spontaneous fracture of plastic biliary and PD stents have been previously reported. ${ }^{9,10}$ The factors responsible for spontaneous fracture of plastic stents are not clear but the 
factors that are presumed to be contributing are the type of stent used, duration of stenting, and surrounding chemical environment of the stent. The spontaneous fractures usually occur at the level of the anchoring flap. In our case series also, the stent fracture was at the level of external pigtail lying in the stomach. Moreover, the acidic gastric environment could also contribute to gradual degradation of the stent materials none of these patients were on proton pump inhibitors.

The mechanism of pain in first two patients is not clear. Although, they presented with pain and pain resolved after removal of stents, it is unlikely that fracture of stents caused pain. The disconnected segment of pancreas has been shown to be having changes suggestive of chronic pancreatitis in $30 \%$ of patients after $>3$ years of follow-up. ${ }^{5}$ It is possible that smoldering pancreatitis in disconnected segment could have led on to pain. Long-term follow-up studies are needed to ascertain the cause of pain in these patients with fractured stents.

In conclusion, spontaneous fracture of permanent indwelling transmural plastic stents is a are complication of permanent indwelling transmural stents. The gastroenterologists should be aware of this rare complication of otherwise safe and effective strategy of leaving transmural plastic stents for an indefinite period in patients of WON with DPDS.

\section{Author Contributions}

1. Surinder Singh Rana: Collection and interpretation of data, as well as drafting of manuscript

2. Ravi Sharma: Collection and interpretation of data

3. Rajesh Gupta: Collection and interpretation of data

\section{Conflicts of Interest}

There are no conflicts of interest and no financial disclosures to be made by any of the authors.

\section{References}

1 Larsen M, Kozarek RA. Management of disconnected pancreatic duct syndrome. Curr Treat Options Gastroenterol 2016;14(3):348-359

2 Rana SS, Bhasin DK, Sharma R, Gupta R. Factors determining recurrence of fluid collections following migration of intended long term transmural stents in patients with walled off pancreatic necrosis and disconnected pancreatic duct syndrome. Endosc Ultrasound 2015;4(3):208-212

3 Rana SS, Bhasin DK, Rao C, Sharma R, Gupta R. Consequences of long term indwelling transmural stents in patients with walled off pancreatic necrosis \& disconnected pancreatic duct syndrome. Pancreatology 2013;13(5):486-490

4 Varadarajulu S, Rana SS, Bhasin DK. Endoscopic therapy for pancreatic duct leaks and disruptions. Gastrointest Endosc Clin N Am 2013;23(4):863-892

5 Rana SS, Shah J, Sharma RK, Gupta R. Clinical and morphological consequences of permanent indwelling transmural plastic stents in disconnected pancreatic duct syndrome. Endosc Ultrasound 2020;9(2):130-137

6 Téllez-Aviña FI, Casasola-Sánchez LE, Ramírez-Luna MÁ, et al. Permanent indwelling transmural stents for endoscopic treatment of patients with disconnected pancreatic duct syndrome: long-term results. J Clin Gastroenterol 2018;52(1):85-90

7 Varadarajulu S, Wilcox CM. Endoscopic placement of permanent indwelling transmural stents in disconnected pancreatic duct syndrome: does benefit outweigh the risks. ? Gastrointest Endosc 2011;74(6):1408-1412

8 Rana SS, Sharma R, Gupta R. Pancreatic parenchymal calcification induced by permanent indwelling transmural stent. JGH Open 2019;3(3):264-265

9 Kawaguchi Y, Lin J-C, Kawashima Y, et al. Risk Factors for Migration, Fracture, and Dislocation of Pancreatic Stents. Gastroenterology Research and Practice. Available at: https:// www.hindawi.com/journals/grp/2015/365457/. Accessed April 26, 2020

10 HCPLive.Using Radiography to DetectaBiliaryStentFractureand Related Bowel Obstruction. Available at: https://www.mdmag. com/journals/surgical-rounds/2008/2008-02/2008-02_03. Accessed April 26, 2020 